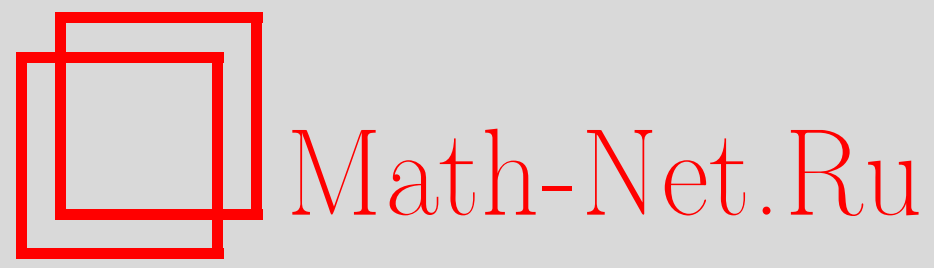

Д. С. Широков, Теорема о норме элементов спинорных групп, Вестн. Сам. гос. техн. ун-та. Сер. Физ.-мат. науки, 2011, выпуск 1(), 165-171

DOI: https://doi.org/10.14498/vsgtu875

Использование Общероссийского математического портала Math-Net.Ru подразумевает, что вы прочитали и согласны с пользовательским соглашением http://www. mathnet.ru/rus/agreement

Параметры загрузки:

IP : 107.22 .136 .117

26 апреля 2023 г., 04:31:31 
УДК 512.5

\section{ТЕОРЕМА О НОРМЕ ЭЛЕМЕНТОВ СПИНОРНЫХ ГРУПП}

\section{Д. С. Широков}

Математический институт им. В. А. Стеклова РАН, 119991, Москва, ул. Губкина, 8.

E-mail: shirokov@mi.ras.ru

Рассмотрена алгебра Клифборда над полем вещественнъх чисел произволъной конечной размерности. Для элементов алгебры Клифборда с фиксированным базисом определяется операчия эрмитова сопряжения, которая позволяет задать структуру евклидова пространства на алгебре Клифборда. Рассмотрень псевдоортогональная группа и её подгруппь - специальная псевдоортогональная, ортохронная, ортохорная и специальная ортохронная. Как известно, рассмотренные 5 групп дважды накрываются соответствующими спинорными группами. Доказана теорема, связывающая норму произволъного элемента спинорной группь с минором матрицы из соответствующей ортогональной группь.

Ключевые слова: алгебра Клиффорда, спинорные группы, ортогоналъные группы, ортохронная группа, ортохорная группа.

Введение. В статье рассмотрена алгебра Клиффорда [1] над полем вещественных чисел произвольной конечной размерности. Алгебра Клиффорда является одним из возможных обобщений вещественных, комплексных чисел и кватернионов и имеет применение во многих разделах современной математики и физики. $\mathrm{K}$ примеру, алгебра Клиффорда находит своё применение в теории поля [2], робототехнике, обработке сигналов и изображений, химии, небесной механике, электродинамике и др.

Для элементов алгебры Клиффорда с фиксированным базисом вводится операция эрмитова сопряжения, согласованная с операцией эрмитова сопряжения матриц. На основе этой операции строится операция скалярного произведения, превращающая алгебру Клиффорда в евклидово пространство.

Рассмотрены псевдоортогональная группа $\mathrm{O}(p, q)$ и её подгруппы - специальная псевдоортогональная $\mathrm{SO}(p, q)$, ортохронная $\mathrm{O}_{\uparrow}(p, q)$, ортохорная $\mathrm{O}_{\downarrow}(p, q)$ и специальная ортохронная $\mathrm{SO}_{\uparrow \downarrow}(1,3)$. Доказана теорема, связывающая главные миноры рассматриваемых ортогональных групп.

Как известно, рассмотренные 5 ортогональных групп дважды накрываются соответствующими спинорными группами $\operatorname{Pin}(p, q), \operatorname{Spin}(p, q), \operatorname{Pin}_{\uparrow}(p, q)$, $\operatorname{Pin}_{\downarrow}(p, q)$ и $\operatorname{Spin}_{\uparrow \downarrow}(p, q)$. Накрытие описывается формулой

$$
S^{\wedge} e^{a} S^{-1}=p_{b}^{a} e^{b},
$$

которая однозначно ставит в соответствие паре элементов $\pm S$ спинорной группы единственную матрицу $P=\left\|p_{b}^{a}\right\|$ из соответствующей ортогональной группы.

Доказана теорема, показывающая, что норма элементов спинорной группы, введенная на основе рассматриваемого скалярного произведения, тесно связана с минорами соответствующей ей ортогональной матрицы.

Дмитрий Сергеевич Широков, аспирант, отд. математической физики. 
1. Алгебры Клиффорда над полем вещественных чисел. В литературе известны несколько различных (эквивалентных) определений алгебр Клиффорда. В рассматриваемом далее определении [2-4] алгебры Клиффорда используется базис специального вида - занумерованный упорядоченными мультииндексами. Подчеркнем, что введённые далее генераторы и базис фиксированы.

Пусть $E$ - векторное пространство над полем вещественных чисел $\mathbb{R} ; n-$ натуральное число, и размерность пространства $E$ равна $\operatorname{dim} E=2^{n}$. Пусть в $E$ введён базис

$$
\left.e, e^{a}, e^{a_{1} a_{2}}, \ldots, e^{1 \ldots n}, \quad \text { где } \quad a_{1}<a_{2}<\ldots \quad \text { (их } 2^{n} \text { штук }\right),
$$

занумерованный упорядоченными мультииндексами длины от 0 до $n$. Индексы $a, a_{1}, a_{2}, \ldots$ пробегают значения от 1 до $n$.

Пусть $p$ и $q$-неотрицательные целые числа и $p+q=n, n \geqslant 1$. Введём диагональную матрицу $\eta=\left\|\eta^{a b}\right\|=\operatorname{diag}(1, \ldots, 1,-1, \ldots,-1)$, у которой на диагонали стоят $p$ штук +1 и $q$ штук -1 .

Введём на $E$ операцию Клифбордова умножения $U, V \rightarrow U V$, по отношению к которой выполнены свойства дистрибутивности, ассоциативности, $e-$ единичный элемент и, кроме того,

$$
\begin{gathered}
e^{a_{1}} \ldots e^{a_{k}}=e^{a_{1} \ldots a_{k}}, \quad 1 \leqslant a_{1}<\ldots a_{k} \leqslant n, \\
e^{a} e^{b}+e^{b} e^{a}=2 \eta^{a b} e, \quad \forall a, b=1, \ldots n .
\end{gathered}
$$

Тогда введённая таким образом алгебра называется вещественной алгеброй Клиффорда и обозначается $\mathcal{C l}^{\mathbb{R}}(p, q)=\mathcal{C} \ell(p, q)$.

Любой элемент $U$ алгебры Клиффорда $\mathcal{C}(p, q)$ представляется в виде разложения по базису (1):

$$
U=u e+u_{a} e^{a}+\sum_{a_{1}<a_{2}} u_{a_{1} a_{2}} e^{a_{1} a_{2}}+\ldots+u_{1 \ldots n} e^{1 \ldots n}
$$

где $u, u_{a}, u_{a_{1} a_{2}}, \ldots, u_{1 \ldots n}-$ вещественные числа.

Понятия РАнгов и чётности. Векторные подпространства, натянутые на элементы $e^{a_{1} \ldots a_{k}}$, занумерованные упорядоченными мультииндексами длины $k$, обозначаются как $\mathcal{C}_{k}(p, q)$. Элементы подпространства $\mathcal{C}_{k}(p, q)$ называются элементами ранга $k$. Имеем

$$
\mathcal{C} \ell(p, q)=\oplus_{k=0}^{n} \mathcal{C} \ell_{k}(p, q) .
$$

Алгебра Клиффорда $\mathcal{C}(p, q)$ является супералгеброй, а именно представляется в виде прямой суммы чётного и нечётного подпространств:

$$
\begin{gathered}
\mathcal{C l}(p, q)=\mathcal{C}_{\text {even }}(p, q) \oplus \mathcal{C} \ell_{\text {odd }}(p, q), \\
\mathcal{C} \ell_{\text {even }}(p, q)=\mathcal{C} \ell_{0}(p, q) \oplus \mathcal{C} \ell_{2}(p, q) \oplus \mathcal{C} \ell_{4}(p, q) \oplus \ldots, \\
\mathcal{C}_{\text {odd }}(p, q)=\mathcal{C} \ell_{1}(p, q) \oplus \mathcal{C}_{3}(p, q) \oplus \mathcal{C}_{5}(p, q) \oplus \ldots
\end{gathered}
$$


ОПЕРАТОРЫ ПРОЕКТИРОВАНИЯ НА ПОДПРОСТРАНСТВА $\mathcal{C}_{k}(p, q)$. Пусть $U \in$ $\mathcal{C}(p, q)$ записано в виде (2). Введём обозначение для линейных операций проектирования на подпространства элементов ранга $k$ :

$$
\langle U\rangle_{k}=\sum_{a_{1}<\cdots<a_{k}} u_{a_{1} \ldots a_{k}} e^{a_{1} \ldots a_{k}} \in \mathcal{C} \ell_{k}(p, q) .
$$

Используя операцию проектирования на одномерное подпространство $\mathcal{C} \ell_{0}$, натянутое на единичный элемент $e$, введём след элемента $U \in \mathcal{C}(p, q)$ :

$$
\operatorname{Tr}(U)=\left.\langle U\rangle_{0}\right|_{e \rightarrow 1} .
$$

Основное свойство операции $\operatorname{Tr}$ состоит в том, что $\operatorname{Tr}(U V)=\operatorname{Tr}(V U)$.

ОПЕРАцИИ СОПРЯжЕНИЯ. ОПределим оПерации соПряжения в $\mathcal{C}(p, q)$ :

$$
U^{\wedge}=\left.U\right|_{e^{a} \rightarrow-e^{a}}, \quad U^{\sim}=\left.U\right|_{e^{a_{1} \ldots a_{r}} \rightarrow e^{a_{r}} \ldots e^{a_{1}} .}
$$

$U^{\wedge}$ называется чётностным сопряжением (grade involution). Имеем

$$
\begin{gathered}
U^{\wedge}=\sum_{k=0}^{n}(-1)^{k}\langle U\rangle_{k} \quad=\langle U\rangle_{0}-\langle U\rangle_{1}+\langle U\rangle_{2}-\langle U\rangle_{3}+\langle U\rangle_{4}-\ldots, \\
U^{\sim}=\sum_{k=0}^{n}(-1)^{\frac{k(k-1)}{2}}\langle U\rangle_{k}=\langle U\rangle_{0}+\langle U\rangle_{1}-\langle U\rangle_{2}-\langle U\rangle_{3}+\langle U\rangle_{4}+\ldots \\
U^{\wedge \wedge}=U, \quad U^{\sim \sim}=U, \quad(U V)^{\wedge}=U^{\wedge} V^{\wedge}, \quad(U V)^{\sim}=V^{\sim} U^{\sim} .
\end{gathered}
$$

2. Структура евклидова пространства на алгебре Клиффорда. На алгебре Клиффорда $\mathcal{C}(p, q)$ можно ввести структуру евклидова пространства. Для этого надо взять линейную операцию $\dagger: \mathcal{C}(p, q) \rightarrow \mathcal{C} \ell(p, q)$ с помощью условий

$$
\left(e^{i_{1} \ldots i_{k}}\right)^{\dagger}=e_{i_{k}} \ldots e_{i_{1}}, \quad \lambda^{\dagger}=\lambda \quad \text { для } \quad \lambda \in \mathbb{R},
$$

где $e_{a}=\eta_{a b} e^{b}$. Операцию $\dagger$ будем называть операцией эрмитового сопряжсения элементов алгебры Клиффорда. Легко видеть, что

$$
(U V)^{\dagger}=V^{\dagger} U^{\dagger}, \quad U^{\dagger \dagger}=U
$$

Теперь скалярное произведение можно задать формулой

$$
(U, V)=\operatorname{Tr}\left(V^{\dagger} U\right)
$$

Для формулы (5) укажем эквивалентные формулы (ниже они обозначены (6), (7) ), пользоваться которыми, как правило, удобнее.

Tеорема (см. [3]). Рассмотрим вещественную алгебру Клиффорда $\mathcal{C}(p, q)$. Тогда операцию эрмитового сопряжения (5) можно задать также одним из следующих эквивалентных способов:

1)

$$
U^{\dagger}=(-1)^{q} e^{n} \ldots e^{p+1} U^{\sim b} e^{p+1} \ldots e^{n},
$$
2)

где b есть операчия четностного сопряжсения $\wedge$, если $q$-нечётное,

$$
U^{\dagger}=e^{p} \ldots e^{1} U^{\sim \sharp} e^{1} \ldots e^{p},
$$

где $\sharp-\wedge$, если $р-$ чётное. 
3. Ортогональные группы. Рассмотрим псевдоортогональную группу и её подгруппу, специальную псевдоортогональную группу,

$$
\begin{gathered}
\mathrm{O}(p, q)=\left\{A \in \operatorname{Mat}(n, \mathbb{R}) \mid A^{\top} \eta A=\eta\right\}, \\
\mathrm{SO}(p, q)=\left\{A \in \operatorname{Mat}(n, \mathbb{R}) \mid A^{\top} \eta A=\eta, \operatorname{det} A=1\right\},
\end{gathered}
$$

где $p$ и $q$-неотрицательные целые числа такие, что $p+q=n, n \geqslant 1$, а $\eta=\left\|\eta^{a b}\right\|=\operatorname{diag}(1, \ldots, 1,-1, \ldots,-1)-$ диагональная матрица, у которой на диагонали стоят $p$ штук +1 и $q$ штук -1 .

Далее принимаем следующее соглашение. Будем рассматривать сигнатуры $(p, q)$ евклидова пространства $V$, где первые $p$ координат являются временными, а последние $q$ - пространственными.

Через $A_{l_{1} \ldots l_{j}}^{k_{1} \ldots k_{i}}$ будем обозначать минор произвольной матрицы $A$, являющийся определителем матрицы, составленной из элементов, стоящих на пересечении строк с номерами $k_{1}, \ldots k_{i}$ и столбцов с номерами $l_{1}, \ldots l_{j}$.

Рассмотрим ортохронную групnу ("ortochronous"), ортохорную (или сохраняющую чётность) группу ("parity preserving" или "ortochorous" в разных источниках) и специальную ортохронную группу

$$
\begin{gathered}
\mathrm{O}_{\uparrow}(p, q)=\left\{A \in \mathrm{O}(p, q) \mid A_{1 \ldots p}^{1 \ldots p}>0\right\}, \quad \mathrm{O}_{\downarrow}(p, q)=\left\{A \in \mathrm{O}(p, q) \mid A_{p+1 \ldots n}^{p+1 \ldots n}>0\right\},(10) \\
\mathrm{SO}_{\uparrow \downarrow}(p, q)=\left\{A \in \mathrm{O}(p, q) \mid A_{1 \ldots p}^{1 \ldots p}>0, \operatorname{det} A=1\right\} .
\end{gathered}
$$

Частным случаем ортогональных групп являются так называемые групnы Лорениа:

$$
\mathrm{O}(1,3), \mathrm{SO}(1,3), \mathrm{O}_{\uparrow}(1,3), \mathrm{O}_{\downarrow}(1,3), \mathrm{SO}_{\uparrow \downarrow}(1,3) .
$$

Некоторые свойства групп $\mathrm{SO}_{\uparrow \downarrow}(p, q), \mathrm{O}_{\uparrow}(p, q), \mathrm{O}_{\downarrow}(p, q)$ рассмотрены в [5] и других источниках.

4. Теорема о связи миноров ортогональной матрицы. Пусть дана квадратная матрица $A$ размера $n$. Будем называть минор $A_{j_{k+1} \ldots j_{n}}^{i_{k+1} \ldots i_{n}}$ дополнительным к минору $A_{j_{1} \ldots j_{k}}^{i_{1} \ldots i_{k}}$. Всегда будет подразумеваться, что $i_{1}<\ldots<i_{k}$, $j_{1}<\ldots<j_{k}, i_{k+1}<\ldots<i_{n}$ и $j_{k+1}<\ldots<j_{n}$.

Следующая теорема говорит о связи дополнительных миноров матрицы $A$ из псевдоортогональной группы $\mathrm{O}(p, q)$. При доказательстве используем утверждение о миноре обратной матрицы (см. [6]).

Теорема. Пусть матрица А принадлежит псевдоортогональной группе $O(p, q)$. Тогда

$$
A_{i_{1} \ldots i_{k}}^{j_{1} \ldots j_{k}}=(-1)^{\left(\sum_{s=1}^{k} i_{s}+\sum_{s=1}^{k} j_{s}+\sum_{i_{s}>p} 1+\sum_{j_{s}>p} 1\right)} \frac{A_{i_{k+1} \ldots i_{n}}^{j_{k+1} \ldots j_{n}}}{\operatorname{det} A}, \quad \text { ¿de } \quad \operatorname{det} A= \pm 1 .
$$

В частности, получаем

$$
\left|A_{i_{1} \ldots i_{k}}^{i_{1} \ldots i_{k}}\right|=\left|A_{i_{k+1} \ldots i_{n}}^{i_{k+1} \ldots i_{n}}\right|, \quad \operatorname{sgn}\left(A_{i_{1} \ldots i_{k}}^{i_{1} \ldots i_{k}}\right) \operatorname{sgn}\left(A_{i_{k+1} \ldots i_{n}}^{i_{k+1} \ldots i_{n}}\right)=\operatorname{sgn}(A) .
$$

Из этих соотношений получаем важное следствие. А именно, что псевдоортогональная группа $\mathrm{O}(p, q)$ устроена следующим образом. 
При $p, q \neq 0$ группа $\mathrm{O}(p, q)$ состоит из четырех связных компонент:

$$
\begin{gathered}
\mathrm{O}(p, q)=\mathrm{SO}_{\uparrow \downarrow}(p, q) \sqcup \mathrm{O}_{\uparrow}^{\prime}(p, q) \sqcup \mathrm{O}_{\downarrow}^{\prime}(p, q) \sqcup \mathrm{SO}^{\prime}(p, q), \\
\mathrm{O}_{\uparrow}(p, q)=\mathrm{SO}_{\uparrow \downarrow}(p, q) \sqcup \mathrm{O}_{\uparrow}^{\prime}(p, q), \quad \mathrm{O}_{\downarrow}(p, q)=\mathrm{SO}_{\uparrow \downarrow}(p, q) \sqcup \mathrm{O}_{\downarrow}^{\prime}(p, q), \\
\operatorname{SO}(p, q)=\operatorname{SO}_{\uparrow \downarrow}(p, q) \sqcup \mathrm{SO}^{\prime}(p, q) .
\end{gathered}
$$

5. Спинорные группы. Рассмотрим гомоморфизм

$$
\hat{a d}: \mathcal{C} \ell^{\times}(p, q) \rightarrow \operatorname{EndC\ell }(p, q),
$$

действующий на группе обратимых элементов алгебры Клиффорда следующим образом $s \mapsto \hat{a d}$, где $\hat{a d} d_{s} x=s^{\wedge} x s^{-1}$ и $x \in \mathcal{C}(p, q)$.

Обозначим через $\mathcal{C} \ell^{\times}$even $(p, q)$ и $\mathcal{C} \ell^{\times}$odd $(p, q)$ множества чётных и нечётных обратимых элементов алгебры Клиффорда соответственно.

Рассмотрим әруппу Липшииа и её специальную подгруппу:

$$
\begin{gathered}
\Gamma^{ \pm}(p, q)=\left\{s \in \mathcal{C} \ell^{\times}{ }_{\text {even }}(p, q) \cup \mathcal{C} \ell^{\times}{ }_{\text {odd }}(p, q) \mid \forall x \in \mathcal{C} \ell_{1}(p, q), s x s^{-1} \in \mathcal{C l}_{1}(p, q)\right\} \\
\Gamma^{+}(p, q)=\left\{s \in \mathcal{C} \ell^{\times}{ }_{\text {even }}(p, q) \mid \forall x \in \mathcal{C} \ell_{1}(p, q), s x s^{-1} \in \mathcal{C} \ell_{1}(p, q)\right\} .
\end{gathered}
$$

На их основе строятся спинорные группы

$$
\begin{aligned}
\operatorname{Pin}(p, q) & =\left\{s \in \Gamma^{ \pm} \mid s^{\sim} s= \pm e\right\}=\left\{s \in \Gamma^{ \pm} \mid s^{\sim \wedge} s= \pm e\right\}, \\
\operatorname{Pin}_{\downarrow}(p, q) & =\left\{s \in \Gamma^{ \pm} \mid s^{\sim} s=+e\right\}, \\
\operatorname{Pin}_{\uparrow}(p, q) & =\left\{s \in \Gamma^{ \pm} \mid s^{\sim \wedge} s=+e\right\}, \\
\operatorname{Spin}(p, q) & =\left\{s \in \Gamma^{+} \mid s^{\sim} s= \pm e\right\}=\left\{s \in \Gamma^{+} \mid s^{\sim \wedge} s= \pm e\right\}, \\
\operatorname{Spin}_{\uparrow \downarrow}(p, q) & =\left\{s \in \Gamma^{+} \mid s^{\sim} s=+e\right\}=\left\{s \in \Gamma^{+} \mid s^{\sim \wedge} s=+e\right\} .
\end{aligned}
$$

Следующее известное утверждение говорит о связи спинорных и соответствующих ортогональных групп.

Теорема (см. [1], [5] и дР.). Гомоморфизм аd осуществляет двойное накрытие ортогональных групп

$$
\mathrm{O}(p, q), \mathrm{SO}(p, q), \mathrm{SO}_{\uparrow \downarrow}(p, q), \mathrm{O}_{\uparrow}(p, q), \mathrm{O}_{\downarrow}(p, q)
$$

соответствующими спинорными группами

$$
\operatorname{Pin}(p, q), \operatorname{Spin}(p, q), \operatorname{Spin}_{\uparrow \downarrow}(p, q), \operatorname{Pin}_{\uparrow}(p, q), \operatorname{Pin}_{\downarrow}(p, q) .
$$

Связь спинорных и ортогональных групп можно явно выразить следующей формулой:

$$
S^{\wedge} e^{a} S^{-1}=p_{b}^{a} e^{b},
$$

которая однозначно ставит в соответствие паре элементов $\pm S$ из соответствующей спинорной группы (13) единственную матрицу $P=\left\|p_{b}^{a}\right\|$ из соответствующей ортогональной группы (12). 
Группа $\operatorname{Pin}(p, q)$ состоит из четырех компонент:

$$
\begin{gathered}
\operatorname{Pin}(p, q)=\operatorname{Spin}_{\uparrow \downarrow}(p, q) \sqcup \operatorname{Pin}_{\uparrow}^{\prime}(p, q) \sqcup \operatorname{Pin}_{\downarrow}^{\prime}(p, q) \sqcup \operatorname{Spin}^{\prime}(p, q), \\
\operatorname{Pin}_{\uparrow}(p, q)=\operatorname{Pin}_{\uparrow}^{\prime}(p, q) \sqcup \operatorname{Spin}_{\uparrow \downarrow}(p, q), \quad \operatorname{Pin}_{\downarrow}(p, q)=\operatorname{Pin}_{\downarrow}^{\prime}(p, q) \sqcup \operatorname{Spin}_{\uparrow \downarrow}(p, q), \\
\operatorname{Spin}(p, q)=\operatorname{Spin}^{\prime}(p, q) \sqcup \operatorname{Spin}_{\uparrow \downarrow}(p, q) .
\end{gathered}
$$

6. Теорема о норме элементов спинорных групп. Пусть на алгебре Клиффорда $\mathcal{C}(p, q)$ задана структура евклидова пространства. А именно, для любых двух элементов $U, V \in \mathcal{C}(p, q)$ определено их скалярное произведение $(U, V)=\operatorname{Tr}\left(U^{\dagger} V\right)$, где $\dagger$ является операцией эрмитова сопряжения. Скалярное произведение естественным образом порождает норму элемента $\|U\|=$ $=\sqrt{\operatorname{Tr}\left(U^{\dagger} U\right)}$.

Следующая теорема устанавливает связь между нормой элемента спинорной группы и минором ортогональной матрицы, соответствующей этому элементу.

Теорема. Пусть элемент алгебры Клифборда $S$ принадлежит группе $\operatorname{Pin}(p, q)$ и пусть при гомоморфизме $\hat{a d}$ элемент $S$ переходит в ортогональную матрииу $A \in \mathrm{O}(p, q)$. Тогда норма элемента $S$ связана с главными минорами этой матрицы $A_{1 \ldots p}^{1 \ldots p}, A_{p+1 \ldots n}^{p+1 \ldots n}$ следующим образом:

$$
\|S\|^{2}=\operatorname{Tr}\left(S^{\dagger} S\right)= \begin{cases}A_{1 \ldots p}^{1 \ldots p}=A_{p+1 \ldots n}^{p+1 \ldots n}, & \text { если } S \in \operatorname{Spin}_{\uparrow \downarrow}(p, q) ; \\ A_{1 \ldots p}^{1 \ldots p}=-A_{p+1 \ldots n}^{p+1 \ldots n}, & \text { если } S \in \operatorname{Pin}_{\uparrow}^{\prime}(p, q) ; \\ -A_{1 \ldots p}^{1 \ldots p}=A_{p+1 \ldots n}^{p+1 \ldots n}, & \text { если } S \in \operatorname{Pin}_{\downarrow}^{\prime}(p, q) ; \\ -A_{1 \ldots p}^{1 \ldots p}=-A_{p+1 \ldots n}^{p+1 \ldots n}, & \text { если } S \in \operatorname{Spin}^{\prime}(p, q) .\end{cases}
$$

Отметим, что выражение $\|U\|^{2}=\operatorname{Tr}\left(U^{\dagger} U\right)>0$ всегда положительно и, таким образом, формулы (14) согласуются с определением ортогональных групп (10).

Утверждение теоремы представляет из себя особую связь ортогональных и спинорных групп, которая, вообще говоря, иллюстрирует также двойные накрытия ортогональных групп спинорными.

Доказанная теорема позволяет, не находя явно элемента $S \in \operatorname{Pin}(p, q)$ спинорной группы, вычислять его норму по соответствующей ему ортогональной матрице $A \in \mathrm{O}(p, q)$.

Автор выражает благодарность за поддержку на конференции «Математическая физика и ее приложения - 2010» лабораторией математической физики СамГУ, грантами АВЦП 3341 и 10854 и контрактом ФЦП 2173.

\section{БИБЛИОГРАФИЧЕСКИЙ СПИСОК}

1. Lounesto P. Clifford algebras and spinors/ L.M.S. Lecture Notes. Vol. 239. Cambridge: Cambridge Univ. Press, 1997. 306 pp.

2. Марчук Н. Г. Уравнения теории поля и алгебры Клиффорда. М.-Ижевск: НИЦ РХД, 2009. 304 c. [Marchuk N. G. Equations of field theory and Clifford algebras. Moscow, Izhevsk: RKhD, 2009. 304 pp.]

3. Marchuk N. G., Shirokov D. S. Unitary spaces on Clifford algebras // Adv. in Appl. Cliff. Alg., 2008. Vol. 18, no. 2. Pp. 237-254. 
4. Широков Д. С. Классификация элементов алгебр Клиффорда по кватернионным типам // ДАН, 2009. Т. 427, №6. С. 758-760; Shirokov D.S. Classification of elements of clifford algebras according to quaternionic types // Doklady Mathematics, 2009. Vol. 80, no. 1. Pp. 610-612.

5. Benn I. M., Tucker R. W. An Introduction to Spinors and Geometry with Applications in Physics. Bristol: IOP Publishing Ltd, 1987. 358 pp.

6. Гантмахер Ф.Р. Теория матриц. М.: Наука, 1988. 549 с. [Gantmakher F. R. Theory of matrices. Moscow: Nauka, 1988. 549 pp.]

MSC: 15A66

\section{THEOREM ON THE NORM OF ELEMENTS OF SPINOR GROUPS}

\section{S. Shirokov}

Steklov Mathematical Institute, Russian Academy of Sciences,

8, Gubkina st., Moscow, 119991, Russia.

E-mail: shirokov@mi.ras.ru

In this article we consider Clifford's algebra over the field of real numbers of finite dimension. We define the operation of Hermitian conjugation for the elements of Clifford's algebra. This operation allows us to define the structure of Euclidian space on the Clifford algebra. We consider pseudo-orthogonal group and its subgroups - special pseudo-orthogonal, orthochronous, orthochorous and special orthochronous groups. As known, spinor groups are double covers of these orthogonal groups. We proved a theorem that relates the norm of element of spinor group with the minor of matrix of the orthogonal group.

Key words: Clifford's algebra, spinor groups, orthogonal groups, orthochronous group, orthochorous group.

Original article submitted 20/12/2010; revision submitted $17 / 02 / 2011$.

Dmitry S. Shirokov, Postgraduate Student, Dept. of Mathematical Physics. 